\title{
Capital intangible y capital intelectual: Revisión, definiciones y líneas de investigación
}

\author{
PEP SIMÓ y JOSÉ MARÍA SALLÁN \\ Departament d'Organització d'Empreses \\ Escola Tècnica Superior d'Enginyeries Industrial i Aeronàutica de Terrassa \\ UNIVERSITAT POLITÈCNICA DE CATALUNYA \\ e-mail: pep.simo@upc.edu; jose.maria.sallan@upc.edu
}

\begin{abstract}
RESUMEN
Dos de los problemas más importantes de la investigación en intangibles y capital intelectual son la falta de una terminología común, y el poco desarrollo de escalas de medición de los constructos asociados a los intangibles. El objetivo de este artículo es doble: en primer lugar, establecer unas definiciones integradas y coherentes de los conceptos más empleados en intangibles, como activos y pasivos intangibles, y capital intelectual; y segundo lugar definir un conjunto de dimensiones para el capital intelectual coherente desde el punto de vista teórico, y que afecte a propiedades relevantes para la gestión del capital intelectual. Después de un análisis longitudinal de la literatura sobre intangibles de las últimas tres décadas, se establece una definición de capital intelectual como conocimiento que crea valor, siendo por tanto un caso particular de activo intangible. Se proponen también dos dimensiones de capital intelectual, asociadas a los conceptos de conocimiento tácito y conocimiento explícito.
\end{abstract}

Palabras clave: Capital intangible; capital intelectual; activos intangibles; pasivos intangibles.

\section{Intangible Capital and Intellectual Capital: Literature Review, Definitions and Agenda for Research}

\begin{abstract}
Two of the most salient issues in research about intangibles and intellectual capital are the lack of a common terminology, and the lack of development of adequate measurement tools for the constructs related to intangibles. The aim of that paper is twofold: first, establishing a set of integrated, coherent definitions for the core concepts in intangibles research, such as intellectual capital or intangible assets and liabilities. The second objective is to define a set of intellectual capital dimensions theoretically coherent and useful for intellectual capital management. Through a longitudinal examination of the most relevant contributions in research on intangibles, we propose a new definition of intellectual capital as knowledge that creates value, being then a subset of the intangible assets of the organization. Finally, we propose two dimensions of intellectual capital, related to the concepts of tacit and explicit knowledge.
\end{abstract}

Keywords: Intangible Capital; Intellectual Capital; Intangible Assets, Intangible Liabilities.

Clasificación JEL: L2, M1, M4.

Artículo recibido en mayo de 2008 y aceptado para su publicación en julio de 2008.

Artículo disponible en versión electrónica en la página www.revista-eea.net, ref. อ-26204. 


\section{INTRODUCCIÓN}

La percepción de la importancia de los intangibles y del capital intelectual en las organizaciones viene de antiguo (Kristandl y Bontis, 2007). La primera mención del concepto de intangible en la empresa, atribuida a Lawrence R. Dicksse, data de 1896 (Wu, 2005), mientras que la primera aparición del término capital intelectual data de 1969, en una comunicación privada del economista John K. Galbraith (Bontis, 1998). Pero es en los años noventa cuando, coincidiendo con el auge de la economía del conocimiento, los consultores en gestión empresarial empiezan a prestar atención a los intangibles y al capital intelectual. En estos años, se desarrollaron no menos de treinta metodologías de evaluación y medición del capital intelectual y/o los intangibles (Andriessen, 2004a), con un gran éxito en el ámbito empresarial: en 2001, el 52,3\% de las empresas cotizadas en la Bolsa de Madrid presentaban información voluntaria sobre su capital humano (Villacorta, 2006). Sin embargo, este éxito no se ha traducido en acumulación de conocimiento (Garcia-Parra, Simo y Sallan, 2006), y algunas de las contribuciones se han caracterizado por su escaso rigor científico.

Dos de los problemas más importantes de la investigación en intangibles son la falta de una terminología común (Kristandl y Bontis, 2007) y el poco desarrollo de escalas de medición de los constructos asociados a los intangibles. Es bastante común que cada autor trate de construir una teoría de intangibles desde el principio, creando nuevas definiciones, introduciendo nuevas dimensiones de intangibles y de capital intelectual, y tratando de imponer su propia terminología (Bontis, 2001). Este hecho dificulta la acumulación de conocimiento, y justifica los escasos progresos que se han realizado en los últimos veinte años en la construcción de una teoría de los intangibles en las organizaciones (Andriessen, 2004b; Bontis, Dragonetti, Jacobsen y Roos, 1999; Molbjerg-Jorgensen, 2006; Sveiby, 1997). La poca consistencia de las terminologías empleadas ha dificultado el establecimiento de mediciones claras de los intangibles, especialmente de las dimensiones del capital intelectual, lo cual ha supuesto una dificultad adicional al progreso en este ámbito del conocimiento.

El primer objetivo de este artículo es contribuir a establecer una terminología consistente para el estudio de los intangibles. Para ello, evaluaremos las definiciones más relevantes de intangibles, capital intelectual, activos intangibles y pasivos intangibles propuestas hasta la fecha, para encontrar una definición consistente e integradora de estos constructos. El segundo objetivo es contribuir a clarificar la identificación y medición de las dimensiones del capital intelectual, con dos finalidades: proponer un conjunto de dimensiones de capital intelectual coherente y consistente, y poner en evidencia la problemática asociada a la medición de este constructo. Pretendemos, por tanto, hacer un primer esbozo de una teoría de los intangibles en las organizaciones, que permita abrir nuevas líneas de investigación con el objetivo de comprender, medir y analizar los fenómenos relacionados con los intangibles en las organizaciones. 


\section{LA EVOLUCIÓN DE LOS INTANGIBLES Y DEL CAPITAL INTELECTUAL}

La revisión de la literatura sobre intangibles pone en evidencia la variedad de denominaciones asignadas a conceptos similares. Para referirse a un concepto similar o idéntico, los contables han usado el término intangibles, los economistas activos de conocimiento y los juristas propiedad intelectual (Lev, 2001). En parte, esta diversidad terminológica se debe a que el estudio de los intangibles se ha tratado desde diversas perspectivas y disciplinas, principalmente la contabilidad y los recursos humanos, empleando estos últimos el término capital intelectual para referirse a un concepto similar, al menos en apariencia (Johanson, 2000). Por tanto, nuestra primera tarea será analizar la evolución de los estudios sobre intangibles desde una perspectiva temporal, integrando las aportaciones de las diferentes disciplinas.

Los trabajos de Petty y Guthrie (2000) y Garcia-Parra et al. (2006) nos dan una perspectiva amplia de cómo ha evolucionado esta área y desde que prisma se ha tratado. Más allá de las primeras citaciones del término, podemos situar el inicial auge de los intangibles y del capital intelectual a finales de los años ochenta y mediados de los noventa del siglo pasado. Con la aparición de la economía del conocimiento y el inicio de la denominada era de la información cobra relevancia encontrar explicaciones para las grandes diferencias existentes entre el valor de mercado y el valor contable de ciertas empresas cotizadas (Garcia-Parra et al., 2006). Es en esta época cuando autores como Edvinsson y Malone (1997) denominan capital intelectual a la diferencia entre el valor de mercado y el valor contable. Durante los años noventa se van desarrollando distintos modelos y aplicaciones para medir y gestionar el capital intelectual (p. ej., Celemi, Skandia Navigator, Intangible Assets Monitor, dirección por competencias, etc.), de los que el más conocido fue el cuadro de mando integral de Kaplan y Norton (1992). Fue en este contexto en el que se designó el primer director de capital intelectual en una empresa: Leif Edvinsson en Skandia AFS (Garcia-Parra et al., 2006).

A principios de este siglo la cuestión de los intangibles empieza a cobrar interés para la comunidad académica. Upton (2001), por ejemplo, critica la definición de capital intelectual como diferencia entre valor contable y valor en libros. Su argumentación se basa en que existen múltiples factores, diferentes del capital intelectual, que pueden explicar esta diferencia, como la sobrevaloración de activos tangibles y financieros, la subvaloración de los pasivos exigibles, cuestiones legales o efectos temporales como el "efecto Enero" (Andriessen, 2004b; Garcia-Ayuso, 2003; Upton, 2001). Es en este momento cuando se empiezan a organizar congresos científicos sobre intangibles, y a desarrollar iniciativas, como el proyecto Meritum (2002), con el objetivo de estandarizar conceptos y taxonomías, introduciendo mayor rigor académico en el estudio de intangibles. Sin embargo, son aún escasos los trabajos empíricos publicados con suficiente rigor que establezcan una definición clara de los intangibles, del capital intelectual y de las posibles dimensiones de éste. La mayoría de literatura publicada sigue siendo de carácter prescriptivo. Este tipo de literatura, creada con perspectiva eminentemente comercial, induce una gran dispersión y su- 
perposición de definiciones, con escaso rigor académico, centrándose en describir la naturaleza de los intangibles, sugiriendo categorizaciones y taxonomías (Kristandl y Bontis, 2007). El resultado, como veremos a continuación, es la proliferación de definiciones de intangibles, activos intangibles, capital intelectual, capital humano, e incluso pasivos intangibles, a veces reiterativas y a veces contradictorias.

\section{REVISIÓN Y DISCUSIÓN DE LAS DEFINICIONES}

Según la vigésimo segunda edición del diccionario de la Real Academia de la Lengua Española, intangible es un adjetivo atribuible a aquello que no se puede tocar, y que carece por tanto de sustancia física. La definición de intangibles en gestión empresarial dista de la concreción de la utilización ordinaria de este término. La mayoría de los autores han definido los intangibles no por lo que son, sino por lo que no son, y otros autores obvian definir el término describiendo directamente su composición o su articulación en dimensiones (Krinstandl y Bontis, 2007). Por ejemplo, Bontis (1996) se refirió al capital intelectual como la captura, codificación y diseminación de la información, para adquirir nuevas competencias para la reingeniería de procesos. Esta definición parece referirse más a los procesos para adquirir capital intelectual, o definir diferentes componentes del capital intelectual, más que al capital intelectual en sí.

Un año después, Stewart (1997), Roos, Roos, Dragonetti y Edvinsson (1997), Edvinsson y Malone (1997) y Sveiby (1997) aportaron nuevas definiciones del capital intelectual y de los activos intangibles. Stewart (1997) definió el capital intelectual como la suma de todo lo que cada uno sabe en la compañía y proporciona a ésta una ventaja competitiva en el mercado. Desde un punto de vista más generalista, Roos et al. (1997) lo definieron como la suma de activos ocultos de una compañía que no están totalmente capturados o representados en el balance de situación; y Edvinsson y Malone (1997) como la posesión de conocimiento, experiencia aplicada, tecnología organizativa, relaciones con los clientes y capacidades profesionales que proveen a la empresa de una ventaja competitiva en el mercado. De manera similar en que Roos et al. (1997) definieron el capital intelectual, Sveiby (1997) definió los activos intangibles como activos invisibles que incluyen la competencia de los empleados, la estructura interna y la externa. Ya en estos primeros años observamos que los términos capital intelectual y activos intangibles son casi intercambiables. Las definiciones de Roos et al. (1997) y Sveiby (1997) incluyeron conceptos no definidos previamente por los autores, como activos invisibles y activos ocultos, que luego son utilizados como sinónimos del capital intelectual o de los intangibles. Por otro lado Stewart (1997) y Edvinsson y Malone (1997), influenciados por la visión basada en recursos (Barney, 1991; Barney, Wright y Ketchen, 2001; Penrose, 1959; Wernerfelt, 1984) incluyeron en el capital intelectual todos aquellos activos que pueden proporcionar ventaja competitiva.

Un año después, en 1998, Sullivan (1998) definió el capital intelectual como el conocimiento que puede ser convertido en beneficios en el futuro. El problema de 
esta definición es que para medir el capital intelectual hemos de esperar a que los beneficios que éste pueda generar se hayan producido y medido. Además, cabe preguntarse porqué el conocimiento actual no puede ser el causante de los beneficios actuales. Para Brooking (1998), el capital intelectual es aquella combinación de activos intangibles con una función en la compañía, y que consisten en activos de mercado, activos de propiedad intelectual, activos humanos y activos de Infraestructura. Para esta autora el capital intelectual es, por tanto, una combinación de activos intangibles, pero no aclara la diferencia entre capital intelectual y activos intangibles. Finalmente, Ulrich (1998), desde un enfoque más próximo a la gestión de los recursos humanos, definió capital intelectual como el producto de competencia por compromiso. El problema de esta definición es que a pesar de disponer de modelos teóricos y herramientas para medir el compromiso y las competencias individuales de forma suficientemente fiable cuando la unidad de análisis es el individuo, resulta mucho más problemático evaluar estos constructos a nivel organizativo. Por tanto, al aplicar la definición de Ulrich, no estaríamos midiendo el capital intelectual de la organización, sino el de cada uno de los individuos que la compone.

El cambio de siglo ha traído pocas novedades a la definición del capital intelectual, pero sí que observamos que empieza a usarse cada vez más el término intangibles. Blair y Wallman (2001) definen los intangibles como aquellos factores de producción no físicos que contribuyen a la producción de bienes y servicios, o de los que los individuos u organizaciones que controlan esos factores esperan obtener beneficios en el futuro. Upton (2001), por su parte, define intangibles como ratios, índices u otras informaciones no representadas en los documentos financieros básicos, y considera activos intangibles a aquellos que no tienen sustancia física o financiera relevante. Estas definiciones de intangible no se diferencian significativamente de las anteriores definiciones de capital intelectual. Además, se siguen definiendo los intangibles a partir de lo que no son, y como constructos difíciles de percibir y medir, pues sólo podemos detectar su presencia a partir de sus consecuencias futuras.

Un año después, el proyecto Meritum (2002), propone definiciones diferenciadas para los intangibles, el capital intelectual y los activos intangibles. Define los intangibles como aquellos recursos no monetarios que pueden proporcionar beneficios económicos futuros, sin sustancia física, controlados o como mínimo influenciados por las compañías como resultado de eventos y transacciones previas, y que pueden o no ser identificados de forma separada de otros activos. El capital intelectual abarca todos los tipos de intangibles, ya sean de propiedad y susceptibles de ser utilizados, o bien captados de manera informal para ser desplegados y movilizados. Por último, los activos intangibles son aquella parte de los intangibles o elementos de capital intelectual que pueden ser reconocidos como activo según los modelos contables vigentes.

Durante esta época se empieza a considerar la posibilidad de tener en cuenta que no todos los intangibles son necesariamente activos (Meritum, 2002). La aparición 
del término pasivo intangible la podemos atribuir a Harvey y Lusch (1999) y a los posteriores trabajos de Caddy (2000), Garcia-Parra, Simo, Mundet y Guzman (2004) y Simo y Garcia-Parra (2004). Los trabajos sobre pasivos intangibles se encuadran en dos corrientes: para unos, el pasivo intangible no es más que una minoración del valor de los activos intangibles (Caddy, 2000; Garcia-Ayuso y Larrinaga, 2004; Lozano y Fuentes, 2005), mientras que la otra corriente, más fiel a la definición contable de pasivo, considera los pasivos intangibles como una obligación asociada a los intangibles (Garcia-Parra et al., 2004; Garcia-Parra et al., 2006; Konar y Cohen, 2001).

Las últimas definiciones publicadas de capital intelectual e intangibles no incluyen, sin embargo, referencias a los pasivos intangibles. Viedma (2007) define el capital intelectual como el conocimiento y otros intangibles que crean o producen valor en el presente, y aquellos conocimientos y otros intangibles que pueden crearlo o producirlo en el futuro. Apoyándose en la teoría de los recursos y capacidades, Kristandl y Bontis (2007) proponen que los intangibles son recursos estratégicos que permiten a las organizaciones crear valor de manera sostenible, y que por tanto no están a disposición de un elevado número de empresas. Éstos conducen hacia un potencial de futuros beneficios, los cuales no pueden ser apropiados por otros y no son negociables ni transferibles al mercado.

En cualquier caso, y a pesar de la confusión terminológica, sí que se observan ciertas diferencias entre capital intelectual e intangibles. Por lo general, el término capital intelectual va asociado a conceptos como conocimiento (Edvinsson y Malone, 1997; Stewart, 1997; Sullivan, 1998, Viedma, 2007), competencias de los trabajadores (Edvinsson y Malone, 1997; Ulrich, 1998) o compromiso (Ulrich, 1998). Algunos autores proponen una definición algo más restrictiva de capital intelectual: el capital intelectual es el conocimiento que produce valor (Viedma, 2007) o producirá valor en el futuro (Sullivan, 1998; Viedma, 2007). Las definiciones que se refieren a la obtención de beneficios futuros plantean un problema de medición: si aceptamos estas definiciones, la evaluación del capital intelectual sólo puede ser retrospectiva, pues sólo podremos evaluarlo una vez se hayan producido estos beneficios futuros. Si quisiéramos evaluar el capital intelectual presente deberíamos considerar todos los conocimientos presentes, pues no podremos diferenciar con certeza cuáles de ellos producirán valor en el futuro, o bien tener en cuenta sólo aquellos recursos inimitables y no sustituibles, y que generan valor en el momento presente. Aceptar esta definición supondría asumir que aquellas organizaciones sin ventajas competitivas claras, no basadas en recursos inimitables y no sustituibles, carecen por completo de capital intelectual.

Por otro lado, los intangibles aluden a un concepto más general y amplio, que incluye a los activos intangibles y a los pasivos intangibles (Garcia-Parra et al., 2006). Los activos intangibles son aquellos que no son materiales ni monetarios (Blair y Wallman, 2001; Kristandl y Bontis, 2007; Lev, 2001; Meritum, 2002; Upton, 2001), mientras que los pasivos intangibles, desde una perspectiva de obligación, se referirían a las obligaciones en relación a terceros de carácter no financiero 
ni tangible, y desde la perspectiva de la minoración a las posibles depreciaciones de los activos intangibles (Garcia-Parra et al., 2006). Tal como ocurre con el capital intelectual, considerar la definición de los intangibles desde la perspectiva de la visión basada en recursos propuesta por Kristandl y Bontis (2007), implicaría presuponer que muchas organizaciones carecerían de activos intangibles y pondrían en duda evidencias como que una patente es un activo intangible.

\section{DEFINICIÓN DE CAPITAL INTELECTUAL, ACTIVOS INTANGIBLES, PASIVOS INTANGIBLES, Y CAPITAL INTANGIBLE}

Después de examinar las aportaciones realizadas hasta la fecha, creemos, al igual que Meritum (2002), que debemos diferenciar entre capital intelectual y activos intangibles. De hecho, el concepto de activo intangible es más amplio que el de capital intelectual. Por ello, de forma similar a Sullivan (1998) y Viedma (2007), proponemos la siguiente definición de capital intelectual:

El capital intelectual es el conocimiento propiedad de la organización (conocimiento explícito) o de sus miembros (conocimiento tácito) que crea o produce valor presente para la organización.

Los activos intangibles han sido definidos según la NIC38/IAS38 como aquellos activos identificables de carácter no monetario y sin apariencia física, que se poseen para ser utilizados en la producción o suministros de bienes y servicios, o para ser arrendados a terceros o para funciones administrativas, y que son identificables y controlados por las empresas como resultados de eventos pasados, y de los que se espera que fluyan beneficios económicos futuros. Desde un punto de vista contable, podemos estar totalmente de acuerdo con esta definición, pero el hecho de que tengan que ser identificables imposibilita que activos intangibles como el propio capital intelectual puedan ser considerados. Por lo que proponemos:

Activos intangibles son aquellos activos no monetarios y sin apariencia fisica que se poseen para ser utilizados en la producción o suministro de bienes y servicios.

Luego el propio capital intelectual sería en sí mismo un activo intangible, aunque no el único. Otros posibles activos intangibles podrían ser la reputación de la empresa, el capital organizativo, el capital relacional, las patentes, etc. Para los pasivos intangibles, proponemos una definición integradora de las dos visiones - minoración y obligación-.

Pasivos intangibles son aquellas obligaciones con los stakeholders de la organización, de carácter no monetario ni fisico, cuya no satisfacción, provoca una depreciación de los activos intangibles de la organización. 
Así pues podemos concluir que el capital intangible es la diferencia entre los activos intangibles y los pasivos intangibles, y que el capital intelectual es un activo intangible más, dentro de la variedad de activos intangibles que coexiste en cualquier organización. Utilizándose el termino en plural intangibles como sinónimo del conjunto de activos y pasivos intangibles de una determinada organización.

\section{Capital intangible $=$ Activos Intangibles - Pasivos Intangibles}

A su vez, debemos matizar que hablar de conocimiento que produce valor es hablar de conocimiento aplicado, es decir en una línea similar a Ulrich (1998), es necesario que para que el conocimiento produzca valor exista la competencia y el compromiso organizativo suficiente para aplicarlo eficiente y eficazmente.

\section{DIMENSIONES DEL CAPITAL INTELECTUAL Y MEDICIÓN DE SUS DIMENSIONES}

Uno de los problemas presentes en los pocos trabajos que han intentado medir de forma rigurosa el capital intelectual es la definición de las dimensiones de éste. Ello se debe, probablemente, a que muchos autores han tratado de definir los intangibles y el capital intelectual sugiriendo categorías y taxonomías sin una definición consistente del constructo. Es decir, cuando se plantea la pregunta de qué es el capital intelectual, muchas veces se responde indicando cuántos tipos diferentes de capital intelectual hay. Es como tratar de explicar qué es un coche, contestando que se compone de ruedas, luces, volante, etc. (Kristandl y Bontis, 2007).

El modelo multidimensional más utilizado y reconocido es el propuesto por Bontis, Know y Richardson (2000) quienes lo dividieron en tres dimensiones: capital humano, estructural, y relacional (Andriessen, 2004a; Bontis et al., 2000; Choo \& Bontis, 2002), y del cual existen algunos estudios empíricos consistentes metodológicamente hablando (Martos, Fernandez-Jardon \& Figueroa, 2008; Moon \& Kym, 2006). Pero otros autores han propuesto dimensiones muy dispares, sin apenas validación empírica. Por ejemplo Brooking (1998), proponía dividirlo en activos de mercado, activos de propiedad intelectual, activos humanos, y activos de infraestructura. Bueno (1998) lo dividía en capital humano, relacional, estructural y tecnológico, y Camison et al. (2000) incluyeron el capital organizativo.

A su vez dentro del modelo de tres componentes propuesto por Bontis et al. (2000), cada una de estas dimensiones se ha dividido en distintos subcomponentes (Moon y Kym, 2006). Dentro del capital humano se proponen subcomponentes tales como la capacidad de los empleados o su satisfacción (Kaplan \& Norton, 1996); el capital relacional se ha incluido la cultura y los procesos organizativos (SaintOnge, 1996), los sistemas de información (Stewart, 1997) y la propiedad intelectual (Brooking, 1998), y en el relacional la subcomponte de clientes o los socios (Knight, 1999). Sin embargo, parece poco riguroso definir subdimensiones de unas dimensiones con escasa validez interna. 
Como ya hemos indicado, la definición de componentes del capital intelectual debe ser relevante desde los puntos de vista teórico y práctico y coherente con la propia definición de capital intelectual. Dado que hemos definido el capital intelectual como conocimiento capaz de crear valor, proponemos definir las componentes de éste a partir de la división del conocimiento organizacional en tácito y explícito (Nonaka, 1995). El conocimiento tácito es aquel conocimiento difícil de formalizar y comunicar, y aplicable sólo a un determinado contexto organizacional. El conocimiento tácito también incluye los modelos mentales y creencias que determinan el comportamiento de los miembros de la organización. El conocimiento explícito, por el contrario, es aquel conocimiento formalmente estructurado, comunicable a través de manuales o cualquier otro medio similar, y aplicable y reutilizable en múltiples situaciones y contextos. Dado que el conocimiento tácito reside en los miembros de la organización, podemos considerar la dimensión de capital humano como aquel conocimiento tácito que aporta valor. El conocimiento explícito, por el contrario, reside en la propia organización, por lo que podemos considerar que el conocimiento explícito que aporta valor es el capital estructural.

La investigación empírica muestra que existe una fuerte relación entre las dimensiones de capital humano y capital relacional (Bontis et al., 2000; Martos et al., 2008; Moon y Kim, 2006). Los resultados de estas investigaciones sugieren que el capital relacional, entendido como el conjunto de relaciones de la organización con agentes externos, depende fundamentalmente de la capacidad relacional de sus miembros, por lo que puede considerarse conocimiento tácito y, según nuestra definición, capital humano. Pero el conocimiento asociado a estos contactos también puede residir en la empresa en forma de bases de datos a las que puede acceder cualquier miembro autorizado de la organización. En este caso, se trataría de conocimiento explícito y formarían parte del capital estructural de la organización.

La división del capital intelectual en capital humano y estructural es relevante para la práctica, pues plantea el problema de la apropiación de las rentas generadas por el conocimiento, y la permanencia de ese conocimiento en la organización. Consideremos el caso de una empresa cuyos contactos con los clientes son propiedad exclusivamente de sus empleados comerciales. Si un determinado comercial abandona la organización, ésta puede verse desprovista de determinado conocimiento tácito sobre el cliente, que hasta el momento formaba parte de su capital humano. Después de la salida del comercial, es probable que la empresa disponga de conocimiento explícito sobre el cliente (p.ej., dirección, persona de contacto, ventas realizadas) que forma parte de su capital estructural. Las empresas pueden protegerse, siquiera parcialmente, de los efectos de la rotación de su personal desarrollando procesos de articulación (Nonaka, 1995) del conocimiento que crea valor. Estos procesos permiten convertir el conocimiento tácito en explícito, y en consecuencia, el capital humano en capital estructural.

La acumulación de conocimiento sobre el capital intelectual exige, además de definiciones rigurosas, establecer herramientas de medición consistentes para su utilización en la investigación empírica. Una aportación importante es la de Bontis 
et al. (2000), en el que se propone un conjunto de escalas de Likert para medir el capital "humano", "relacional" y "estructural" de 20, 16 y 17 ítems respectivamente. Estas escalas presentaron un elevado grado de consistencia interna (coeficientes alfa de Cronbach superiores a 0,7) para una muestra de 107 estudiantes de MBA con experiencia laboral no necesariamente gerencial. Sin embargo, el análisis del contenido de estas escalas muestra que incluyen aspectos muy dispares. Por ejemplo, en la escala de capital humano se incluían ítemes donde se pregunta si los empleados de la organización son brillantes, junto con otras en las que se les plantea si se tiene en cuenta la opinión de éstos. La disparidad de contenidos en los ítemes de estas escalas nos lleva a dudar de la validez psicométrica de éstas (Morales, Ullosa y Blanco, 2003), puesto que pueden estar midiendo constructos distintos y con diferentes niveles de intensidad.

Otros estudios empíricos analizados presentan problemas similares, cuando no más graves, de orden metodológico. Así, las escalas de Moon y Kim (2006) para medir las dimensiones de Bontis et al. (2000) de capital intelectual presentan bajos niveles de consistencia interna, con alfas de Cronbach inferiores a 0,5. La investigación de Martos et al. (2008) parte de escalas diferentes, presentando problemas de consistencia interna similares a los de la investigación de Bontis. Estas tres investigaciones evaluaron propiedades organizativas a partir de la información suministrada por un único participante de cada organización, empleados en el estudio de Bontis et al. (2000), gerentes en los de Moon y Kim (2006) y Martos et al. (2008). Los sesgos asociados al hecho de tener un solo informante pueden evitarse o controlarse con estudios multi-informante, en los que se cuenta con varios informantes para cada organización.

En definitiva, podemos concluir que son escasas las investigaciones con cierto rigor en torno a las escalas de medición del capital intelectual, por lo que queda mucho camino por recorrer en esta dirección. El siguiente paso es construir herramientas de medición para evaluar las dimensiones de capital intelectual propuestas, y seguidamente profundizar en el estudio de los antecedentes del capital intelectual, mediante investigaciones de carácter longitudinal.

\section{CONCLUSIONES Y LÍNEAS FUTURAS DE INVESTIGACIÓN}

El análisis longitudinal de los estudios sobre intangibles y capital intelectual muestra que la mayoría de las contribuciones iniciales sobre estas cuestiones vinieron del campo de la consultoría, respondiendo a la preocupación de los directivos por los problemas legales y de naturaleza directiva asociados a los intangibles. Cada una de estas contribuciones iniciales propuso su propia caracterización de los intangibles y el capital intelectual, dando lugar a una gran dispersión conceptual que ha impedido la profundización en el estudio científico de los fenómenos ligados a los intangibles. Creemos que los intangibles son una cuestión fundamental para las organizaciones, y que debe ser estudiada con el adecuado rigor científico. Por ello, 
nuestro primer objetivo ha sido delimitar, mediante un conjunto integrado de definiciones, los significados de los diferentes conceptos utilizados al hablar de intangibles.

En la figura 1 se muestra de manera sintética las relaciones entre estos conceptos. Si definimos el capital intelectual como aquel conocimiento que produce valor, éste formará parte de los activos intangibles de la organización, junto con otros activos intangibles, como la reputación, patentes, entre otros. La contrapartida de los activos intangibles son los pasivos intangibles, definidos como las obligaciones no monetarias con los stakeholders de la organización. El capital intangible de la organización es, entonces, la diferencia entre los activos y los pasivos intangibles. La segunda contribución del artículo ha sido clarificar la cuestión relacionada con las dimensiones del capital intelectual. Nuestra propuesta simplifica la clasificación de Bontis et al. (2000) de capital humano, relacional y estructural, considerando sólo dos dimensiones de capital intelectual: el capital humano, asociado al conocimiento tácito, y el capital estructural, asociado al conocimiento explícito.

FIGURA 1

Modelo conceptual propuesto sobre los intangibles, activos intangibles, pasivos intangibles y capital intelectual.

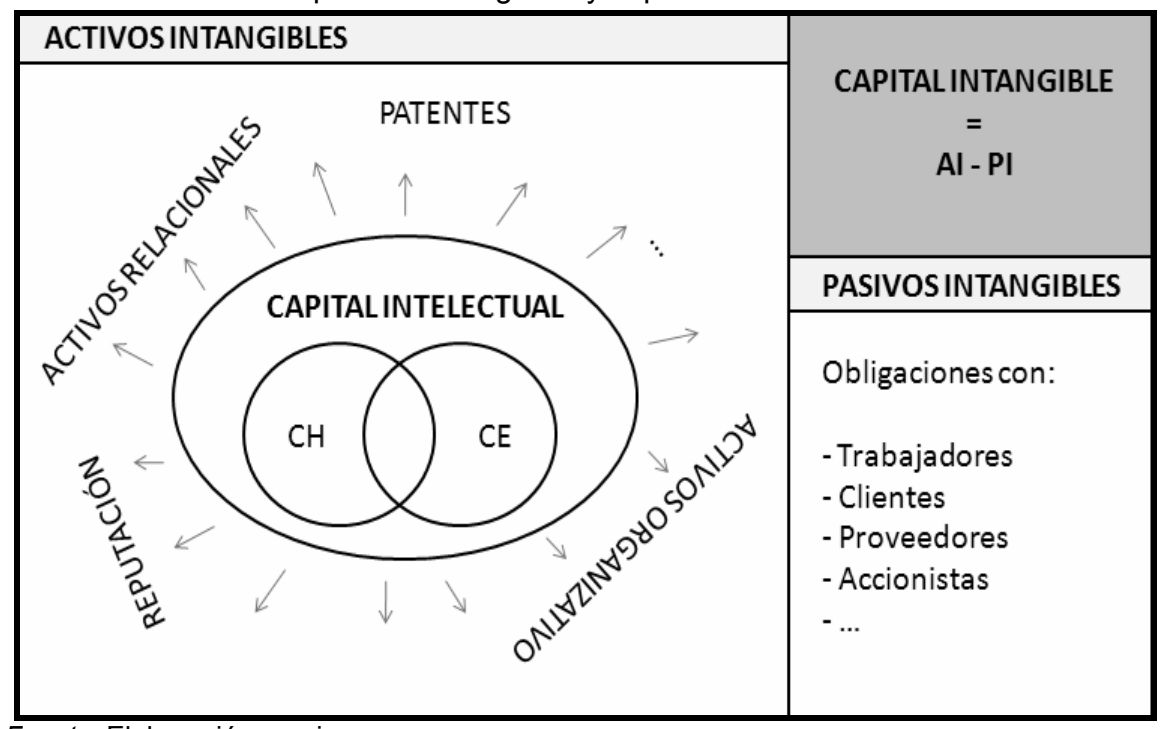

Fuente: Elaboración propia.

La aceptación de las definiciones y clasificaciones aquí propuestas abre varias vías a futuras investigaciones. En primer lugar, es necesario desarrollar metodologías de evaluación del conocimiento tácito y explícito que crean valor en las organizaciones, para medir y evaluar su capital intelectual. Si podemos medir el capital intelectual, estaremos en disposición de evaluar sus antecedentes, y su relación con sus resultados, muchas veces otros activos intangibles. Otra línea de investi- 
gación interesante es preguntarnos por las condiciones en que las organizaciones pueden apropiarse del conocimiento de sus empleados, para convertir el capital humano en capital estructural. Finalmente, es preciso definir taxonomías más precisas que las existentes actualmente para los activos y los pasivos intangibles, que nos ayudarán a avanzar en el conocimiento sobre los procesos con que las organizaciones crean, explotan y obtienen valor de los intangibles.

\section{REFERENCIAS BIBLIOGRÁFICAS}

ANDRIESSEN, D. (2004a): "IC valuation and measurement: Classifying the state of the art" en Journal of Intellectual Capital, $\mathrm{n}^{\circ}$ 5(2), pp. 230-242.

ANDRIESSEN, D. (2004b): Making sense of intellectual capital, Elsevier, Amsterdam.

BARNEY, J. B. (1991): "Firm resources and sustained competitive advantage" en Journal of Management, $\mathrm{n}^{\circ} 17(1)$, pp. 99-120.

BARNEY, J. B.; WRIGHT, M. y KETCHEN, D. J. (2001): "The resource-based view of the firm: Ten years after 1991" en Journal of Management, $n^{\circ} 27(6)$, pp. 625-641.

BLAIR, M. M.; y WALLMAN, S. M. H. (2001): Unseen wealth: Report of the Brookings task force on intangibles, DC: The Brookings Institution, Washington.

BONTIS, N. (1996): "There's a price on your head: managing intellectual capital strategically" en Business Quarterly, Summer, pp. 40-47.

BONTIS, N. (1998): "Intellectual capital: An exploratory study that develops measure and models" en Management Decision, $\mathrm{n}^{\circ}$ 36(2), pp. 63-76.

BONTIS, N. (2001): "Assessing knowledge assets: A review of the models used to measure intellectual capital" en International Journal of Management Reviews, $n^{\circ} 3(1)$, pp. 41-60.

BONTIS, N.; DRAGONETTI, N.; JACOBSEN, K. y ROOS, G. (1999): "The knowledge toolbox: A review of the tools available to measure and manage intangible resources" en European Management Journal, $\mathrm{n}^{\circ}$ 17(4), pp. 391-402.

BONTIS, N.; KEOW, W. C. C. y RICHARDSON, S. (2000): "Intellectual capital and business performance in Malaysian industries" en Journal of Intellectual Capital, $n^{\circ} 1(1)$, pp. 85-100.

BROOKING, A. (1998): Intellectual capital, International Thomson Business Press, London.

BUENO, E. (1998): Medición del capital intelectual: Modelo Intelect, Euroforum, IUEE, San Lorenzo de El Escorial, Madrid.

CADDY, I. (2000): "Intellectual capital: recognizing both assets and liabilities" en Journal of Intellectual Capital, $\mathrm{n}^{\circ} 2$, pp. 129-146.

CAMISON, C.; PALACIOS, D. y DEVECE, C. (2000): "Un nuevo modelo para la medición del capital intelectual: El modelo Nova", Comunicación presentada en el x Congreso de ACEDE, Oviedo.

CHOO, C. W. y BONTIS, N. (2002): The strategic management of intellectual capital and organizational knowledge, NY: Oxford University Press, New York.

EDVINSSON, L. y MALONE, M. S. (1997): Intellectual Capital: Realizing your company's true value by finding its hidden brainpower, NY: Oxford University Press, New York.

GARCIA-AYUSO, M. (2003): "Factors explaining the inefficient valuation of intangibles" en Accounting, Auditing y Accountability Journal, no 16(1), pp. 57-69.

GARCIA-AYUSO, M. y LARRINAGA, C. (2004): "El lado oculto de los intangibles: activos y pasivos ligados a la sostenibilidad" en Revista Finanzas y Contabilidad, n 57, pp. 34-41.

GARCIA-PARRA, M.; SIMO, P.; MUNDET, J. y GUZMAN, J. (2004): "Intangibles: Assets and liabilities" en Intangible Capital, $\mathrm{n}^{\circ} 0(3)$, pp. 70-86.

GARCIA-PARRA, M.; SIMO, P. y SALLAN, J. M. (2006): "La evolución del capital intelectual y las nuevas corrientes" en Intangible Capital, $\mathrm{n}^{\circ}(3)$, pp. 277-307. 
HARVEY, M. G. y LUSCH, R. F. (1999): "Balancing the intellectual capital books: intangible liabilities" en European Management Journal, n 17, pp. 85-92.

JOHANSON, U. (2000): "Characteristics of intangibles: Proposals generated from literature and experienced Swedish firms", en J. E. a. S. Gröjer, H. (Ed.), Classification of Intangibles, Groupe HEC, Paris, pp. 56-77.

KAPLAN, R. S. y NORTON, D. P. (1992): "The balanced scorecard: Measures that drive performance" en Harvard Business Review, $\mathrm{n}^{\circ}$ 70(1), pp. 71-79.

KAPLAN, R. S. y NORTON, D. P. (1996): The Balanced Scorecard: Translating strategy into action, Boston, MA, Harvard Business School.

KNIGHT, D. J. (1999): "Performance measures for increasing intellectual capital" en Strategy and Leadership, $\mathrm{n}^{\circ}$ 29(1), pp. 22-28.

KONAR, S. y COHEN, M. A. (2001): "Does the market value environmental performance?" en Review of Economics and Statistics, $\mathrm{n}^{\circ}$ 83, pp. 281-289.

KRISTANDL, G. y BONTIS, N. (2007): "Constructing a definition for intangibles using the resource based view of the firm" en Management Decision, $n^{\circ}$ 45(9), pp. 1510-1524.

LEV, B. (2001): Intangibles: Management, measurement and reporting. Washington, DC, The Brookings Institute.

LOZANO, M. C. y FUENTES, F. (2005): "La importancia del intangible en la empresa de internet: una propuesta de medición contable" en Economic Analysis Working Papers, $\mathrm{n}^{\circ} 4$, pp. 1-62.

MARTOS, M. S.; FERNANDEZ-JARDON, C. M. y FIGUEROA, P. F. (2008): "Evaluación y relaciones entre las dimensiones del capital intelectual: El caso de la cadena de la madera de Oberá (Argentina)" en Intangible Capital, n 4(2), pp. 67-101.

MERITUM (2002): Directrices para la gestión y difusión de información sobre intangibles: Informe de Capital Intelectual, Fundación Airtel Movil.

MOLBJERG-JORGENSEN, K. (2006): "Conceptualising intellectual capital as a language game and power" en Journal of Intellectual Capital, $n^{\circ} 7(1)$, pp. 78-92.

MOON, Y. J. y KYM H. G. (2006): "A model for the value of intellectual capital" en Canadian Journal of Administrative Sciences, $\mathrm{n}^{\circ}$ 23(3), pp. 253-269.

MORALES, P.; UROSA, B.; BLANCO, A. (2003): Construcción de escalas de actitudes de tipo Likert, La Muralla, Madrid.

NONAKA, I. (1995): The knowledge-creating company: How Japanese companies create the dynamcis of innovation, Oxford University Press, New York.

PENROSE, E. T. (1959): The theory of the growth of the firm, New York, NY: John Wiley.

PETTY, R. y GUTHRIE, J. (2000): "Intellectual capital literature review: Measurement, reporting and management" en Journal of Intellectual Capital, $\mathrm{n}^{\circ} 1(2)$, pp. 156-176.

ROOS, G.; ROOS, J.; DRAGONETTI, N. y EDVINSSON, L. (1997): Intellectual capital: Navigating in the new business landscape, NY: New York University Press, New York.

SAINT-ONGE, H. (1996): "Tacit knowledge: The key to the strategic alignment of intellectual capital" en Strategy y Leadership, $\mathrm{n}^{\circ}$ 24(2), pp. 10-14.

SIMO, P. y GARCIA-PARRA, M. (2004): "Pasivos humanos: antítesis del capital intelectual" en Intangibles, $\mathrm{n}^{\circ} 4$, pp. 24-29.

STEWART, T. A. (1997): Intellectual capital: The new wealth of organizations, NY: Currency Doubleday, New York.

SULLIVAN, P. H. (1998): Profiting from intellectual capital: Extracting value from innovation, NY, Wiley, New York.

SVEIBY, K. E. (1997): The new organisational wealth: Managing and measuring knowledgebased assets, San Francisco, CA, Berrett-Koehler.

ULRICH, D. (1998): "Intellectual capital equals competence x commitment" en Sloan Management Review, $\mathrm{n}^{\circ}$ 39(2), pp. 15-+.

UPTON, W. S. (2001): Business and financial reporting: Challenges from the new economy, Norwalk, CT, FASB. 
VIEDMA, J. M. (2007): "In search of an Intellectual Capital comprehensive theory" en Electronic Journal of Knowledge Management, $n^{\circ} 5(2)$, pp. 245-256.

VILLACORTA, M. A. (2006): "Revelación de la información voluntaria sobre el capital humano en los informes anuales" en Intangible Capital, $\mathrm{n}^{\circ} 2(1)$, pp. 37-71.

WERNERFELT, B. (1984): "A resource-based view of the firm" en Strategic Management Journal, $n^{\circ} 5(2)$, pp. 171-180.

WU, A. (2005): "The integration between balanced scorecard and intellectual capital" en Journal of Intellectual Capital, nº 6(2), pp. 267-284. 